\title{
Timing of endoscopy for acute upper gastrointestinal bleeding: journal club review
}

\author{
Andrew Guy ${ }^{1,2,4} \cdot$ Kate Eppler ${ }^{1,2,4} \cdot$ Jessica Moe ${ }^{1,3}$
}

Received: 22 September 2021 / Accepted: 5 November 2021 / Published online: 25 November 2021

(c) The Author(s), under exclusive licence to Canadian Association of Emergency Physicians (CAEP)/ Association Canadienne de Médecine d'Urgence (ACMU) 2021

Keywords Upper gastrointestinal bleed · UGIB · Endoscopy

Full Citation: Lau J, et al. Timing of Endoscopy for Acute Upper Gastrointestinal Bleeding. New England Journal of Medicine. 2020 Apr 02;382(14):1299-1308

Abstract Link: https://www.nejm.org/doi/pdf/10.1056/ NEJMoa1912484

Article Type: Randomized Controlled Trial

Ratings: Methods—4/5, Usefulness-4/5

\section{Introduction}

\section{Background}

Acute upper gastrointestinal bleeding (UGIB) has a mortality of $10 \%$. Endoscopy within $24 \mathrm{~h}$ is recommended by international consensus guidelines [1] to facilitate treatment of actively bleeding or high-risk lesions. However, data are conflicting on whether earlier endoscopic intervention in high-risk patients with UGIB improves outcomes.

Andrew Guy

andrew.guy@alumni.ubc.ca

1 Department of Emergency Medicine, University of British Columbia, Vancouver, BC, Canada

2 RCPS Emergency Medicine Residency Program, University of British Columbia, Vancouver, BC, Canada

3 Vancouver General Hospital Emergency Department, Vancouver, BC, Canada

4 Royal Columbian Hospital Emergency Department, 330 E Columbia St, New Westminster, BC V3L 3W7, Canada

\section{Objectives}

To evaluate a strategy of urgent $(<6 \mathrm{~h})$ compared to early (6-24 h) endoscopy after gastroenterology consultation for high-risk patients with UGIB.

\section{Methods}

\section{Design}

Prospective, randomized, unblinded, controlled trial.

\section{Setting}

Adult ( $\geq 18$ years) emergency department or medical ward patients in a university hospital in Hong Kong from 2012 to 2018 .

\section{Subjects}

UGIB (melena, hematemesis, or both) and predicted to be at high risk for further bleeding or death (Glasgow-Blatchford 
score $\geq 12$ ) [2]. Patients with shock refractory to initial resuscitation were excluded. Participants otherwise received standard therapy for UGIB (e.g., proton-pump inhibitor bolus and infusion).

\section{Intervention}

Urgent ( $<6$ h) compared to early (6-24 h) endoscopy after gastroenterology consultation.

\section{Outcomes}

Primary outcome was 30-day all-cause mortality. Secondary outcomes included receipt of endoscopic intervention, further bleeding, length of stay in hospital and ICU, need for blood transfusion, need for emergency surgery or angioembolization.

\section{Main results}

516 patients meeting eligibility criteria were enrolled in the trial with 258 assigned to either urgent or early endoscopy. Baseline clinical and demographic variables were similar between the groups, with similar time from presentation to gastroenterology consultation. Time from gastroenterology consultation to endoscopy was $2.5 \pm 1.7 \mathrm{~h}$ and $16.8 \pm 6.8 \mathrm{~h}$ in the urgent and early endoscopy groups, respectively. Emergency endoscopy was performed in 20 patients (7.8\%) in the early endoscopy group.

Outcomes were assessed using an intention-to-treat analysis. The primary outcome of all-cause mortality at 30 days was not significantly different: 23 patients $(8.9 \%)$ in the urgent endoscopy group and $17(6.6 \%)$ in the early endoscopy group died (hazard ratio $1.35 ; 95 \%$ CI $0.72-2.54$ ), with a difference of $2.3 \%$ (95\% CI -2.3 to 6.9 ). There were no statistically significant differences in secondary outcomes, although there was a higher endoscopic intervention rate in the urgent endoscopy group.

\section{Appraisal}

\section{Strengths}

- Clear and clinically important question with significant resource implications.

- Pragmatic study—addresses the presentation of "UGIB" rather than a diagnosis.

- Uses a validated risk-assessment score.
- Patient-centred, objective outcomes.

- Well balanced baseline populations.

- Intention-to-treat analysis.

\section{Limitations}

- Low observed mortality (6.6-8.9\%) compared to their estimate of $16 \%$ used for power calculations.

- $7.8 \%$ of patients in the early endoscopy group received emergency endoscopy: may have diluted any comparative effectiveness in the urgent group.

- Limited generalizability.

- Findings cannot be applied to hemodynamically unstable upper GI bleed patients as these patients were excluded.

- Study performed only in Hong Kong.

- Limited applicability to EDs.

- Timing of endoscopy defined as time from gastroenterology consultation versus time from ED presentation, making comparison to previous studies difficult.

- Unclear why a Glasgow Blatchford score of $\geq 12$ was used, when the authors specifically note that a score $\geq 7$ has been validated in the literature.

\section{Context}

Previous randomized trials have not focused on patients at high-risk for further bleeding or death, and have lacked riskstratification in UGIB. However, it is the opinion of our local experts in gastroenterology that the current trial focusing on high-risk patients is congruent with the results of these prior trials. They also note that perhaps a specific subset of less stable patients would benefit from early endoscopy, but given the ethical considerations involved it would be difficult to design a trial that randomizes these patients to a conservative management strategy.

\section{Bottom line}

This study demonstrated no difference in all-cause mortality at 30 days with a strategy of urgent compared to early endoscopy for high-risk, stable patients with UGIB. While it may be safe to perform endoscopy in a non-urgent manner in this population, patients demonstrating clinical deterioration or with signs of further bleeding should continue to receive emergent endoscopy. 
Acknowledgements We would like to thank Dr. Ian Gan, gastroenterologist at Vancouver General Hospital, BC CANADA for his contributions to this manuscript.

\section{References}

1. Barkun AN, Almadi M, Kuipers EJ, et al. Management of nonvariceal upper gastrointestinal bleeding: guideline recommendations from the International Consensus Group. Ann Intern Med. 2019;171(11):805-22. https://doi.org/10.7326/M19-1795.

2. Stanley AJ, Laine L, Dalton HR, et al. Comparison of risk scoring systems for patients presenting with upper gastrointestinal bleeding: international multicentre prospective study. BMJ. 2017;356: i6432. https://doi.org/10.1136/bmj.i6432. 